\title{
THE DYNAMICS OF MORSE-SMALE DIFFEOMORPHISMS ON THE TORUS \\ BY
}

STEVE BATTERSON

\begin{abstract}
For orientation preserving diffeomorphisms on the torus necessary and sufficient conditions are given for an isotopy class to admit a Morse-Smale diffeomorphism with a specified periodic behavior.
\end{abstract}

The Morse-Smale diffeomorphisms have been shown to be exactly the structurally stable diffeomorphisms with finite nonwandering set [8]. Several recent papers have analyzed the relationship between the dynamics of a Morse-Smale diffeomorphism and the topology of the manifold on which it is defined ([2], [3], [6], and [11]). In [2] Franks related the periodic behavior of a Morse-Smale diffeomorphism to the action of the map on homology. For a given manifold and homotopy (or isotopy) class of maps on that manifold, this result provides a necessary condition for the Morse-Smale dynamics which can occur in the homotopy (isotopy) class. In [0] Narasimhan showed that in the homotopy class of the identity on a compact surface, a given set of Morse-Smale dynamics can be realized provided it satisfies Franks' condition along with two other necessary conditions. We shall prove this result for orientation preserving isotopy classes on the torus $\left(T^{2}=S^{1} \times S^{1}\right)$.

THEOREM. Let $g \in \operatorname{Diff}\left(T^{2}\right)$ and suppose $g$ preserves orientation. There exists a Morse-Smale diffeomorphism $f$ on $T^{2}$ isotopic to $g$ and with peridoic data $\left\{\left(p_{i}, u_{i}, \Delta_{i}\right)\right\}_{i=1}^{n}$ if and only if

(1) $u_{i}=0$ and $u_{j}=2$ for some $i$ and $j$;

(2) $\eta_{g}(t)=\prod_{i=1}^{n}\left(1-\Delta_{i} t^{P_{i}}\right)^{(-1)^{\omega_{i}+1}}$;

(3) $\Delta_{i}=+1$ for each $i$ with $u_{i} \in\{0,2\}$.

I would like to thank John Franks and Ken Mandelberg for valuable conversations and suggestions.

Definitions and background. Let $\operatorname{Diff}(M)$ be the space of diffeomorphisms on a compact manifold $M$. A point $x \in M$ is a nonwandering point of $f \in \operatorname{Diff}(M)$ provided that for any neighborhood $U$ of $x$ there exists a nonzero integer $m$ such that $f^{m}(U) \cap U \neq \varnothing$. The set of nonwandering points of $f$ is denoted $\Omega(f)$. Two diffeomorphisms $f, g \in \operatorname{Diff}(M)$ are topologi-

Received by the editors August 22, 1978 and, in revised form, November 22, 1978. AMS (MOS) subject classifications (1970). Primary 58F20; Secondary 55C20. 
cally (differentiably) conjugate if and only if there exists a homeomorphism (diffeomorphism) $h: M \rightarrow M$ such that $h f=g h$. A diffeomorphism is structurally stable provided there exists a neighborhood $U$ of $f$ in $\operatorname{Diff}(M)$ such that each $g \in U$ is topologically conjugate to $f$.

Suppose $f \in \operatorname{Diff}(M)$ and $f^{m}(x)=x$. Then $x$ is a hyperbolic periodic point of $f$ if the derivative of $f^{m}$ at $x, D f_{x}^{m}$, has spectrum disjoint from the unit circle. If all the eigenvalues of $D f_{x}^{m}$ lie inside the unit circle, then $x$ is called a sink. When each of the eigenvalues has modulus greater than one, $x$ is called a source. Otherwise $x$ is a saddle.

If $\gamma$ is a hyperbolic periodic orbit of period $m$, then for each $x \in \gamma$ let $E_{x}^{u}$ denote the subspace of $T M_{x}$ spanned by the eigenspaces of $D f_{x}^{m}$ corresponding to eigenvalues whose moduli are greater than one. Let $E_{x}^{s}$ be the subspace of $T M_{x}$ spanned by the remaining eigenspaces. Define the orientation type $\Delta$ of $\gamma$ to be +1 if $D f_{x}^{m}: E_{x}^{u} \rightarrow E_{x}^{u}$ preserves orientation and -1 if it reverses orientation. Note that $\Delta$ is well defined.

Suppose $d$ is a metric on $M$ and $p$ is a hyperbolic fixed point of $f$. The stable manifold of $p$ is

$$
W^{s}(p)=\left\{x \in M \mid d\left(p, f^{n}(x)\right) \rightarrow 0 \text { as } n \rightarrow \infty\right\}
$$

and the unstable manifold of $p$ is

$$
W^{u}(p)=\left\{x \in M \mid d\left(p, f^{-n}(x)\right) \rightarrow 0 \text { as } n \rightarrow \infty\right\} .
$$

For a hyperbolic periodic point $p$ of period $m$, the stable and unstable manifolds are defined to be the stable and unstable manifolds of $p$ under $f^{m}$.

Definition. A diffeomorphism is Morse-Smale provided it satisfies the following conditions:

(1) $\Omega(f)$ is finite;

(2) all periodic points are hyperbolic;

(3) for each $p, q \in \Omega(f), W^{s}(p)$ and $W^{u}(q)$ have transversal intersection.

The first condition implies that $\Omega(f)$ is the set of periodic points. Palis and Smale prove in [8] that the Morse-Smale diffeomorphisms are exactly the structurally stable diffeomorphisms with finite nonwandering set. Our goal is to determine precisely the nature of this nonwandering set. by

DefINITION. The homology zeta function $\eta_{f}$ of a diffeomorphism $f$ is defined

$$
\eta_{f}(t)=\prod_{k=0}^{\operatorname{dim} M} \operatorname{det}\left(I-f_{* k} t\right)^{(-1)^{k+1}}
$$

where $f_{* k}: H_{k}(M, R) \rightarrow H_{k}(M, R)$ is the induced map on the $k$ th homology.

Suppose $f$ is a Morse-Smale diffeomorphism. For each periodic orbit $\gamma_{i}$ we associate a triple $\left(p_{i}, u_{i}, \Delta_{i}\right)$ where $p_{i}$ is the period of $\gamma_{i}, u_{i}$ is the dimension of $E_{x}^{u}$ for $x \in \gamma_{i}$, and $\Delta_{i}$ is the orientation type of $\gamma_{i}$. For a diffeomorphism with 
a nonwandering set consisting of a finite number of hyperbolic periodic points, Narasimhan defines the periodic data to be the collection of triples $\left(p_{i}, u_{i}, \Delta_{i}\right)$ ranging over the set of periodic orbits.

THEOREM [0]. Let $M$ be a compact, connected 2-manifold. There exists a Morse-Smale diffeomorphism $f$ of $M$ homotopic to the identity with periodic data $\left\{\left(p_{i}, u_{i}, \Delta_{i}\right) \mid i=1, \ldots, n\right\}$ if and only if:

(a) $u_{i}=0$ and $u_{j}=2$ for some $i$ and $j$;

(b) $\prod_{i=1}^{n}\left(1-\Delta_{i} t^{p_{i}}\right)^{(-1)^{s_{i}+1}}$ is the homology zeta function of the identity;

(c) $\Delta_{i}=+1$ for each $i$ with $u_{i} \in\{0,2\}$.

The necessity of condition (b) is a special case of the following theorem of Franks.

TheOREM [2]. Let $f: M \rightarrow M$ be a $C^{1}$ map with a finite number of periodic orbits all of which are hyperbolic. Then $\eta_{f}(t)=\Pi_{i}\left(1-\Delta_{i} t^{p_{i}}\right)^{(-1)^{\omega_{i}+1}}$.

In this paper we will prove Narasimhan's theorem for orientation preserving isotopy classes on the torus. Shub has shown [10] that for a homotopy class to admit a Morse-Smale diffeomorphism the induced homology maps must be quasi-unipotent (i.e., every eigenvalue is a root of unity). For $T^{2}$ this condition is also sufficient.

We close this section by stating two lemmas from [6].

LEMMA 1. If

$$
\frac{\Pi_{i=1}^{m}\left(1-t^{q_{i}}\right) \Pi_{i=1}^{n}\left(1+t^{r_{i}}\right)}{\Pi_{j=1}^{l}\left(1-t^{p_{J}}\right)}=1
$$

then

(a) $m=l$;

(b) there exist integers $a_{1}>0, \ldots, a_{m} \geqslant 0$ such that

(i) the set $\left\{p_{1}, p_{2}, \ldots, p_{m}\right\}$ equals the set $\left\{2^{a_{1}} q_{1}, 2^{a_{2}} q_{2}, \ldots, 2^{a_{m}} q_{m}\right\}$;

(ii) the set $\left\{r_{1}, r_{2}, \ldots, r_{n}\right\}$ equals the set

$\left\{q_{1}, 2 q_{1}, \ldots, 2^{a_{1}-1} q_{1} ; q_{2}, 2 q_{2}, \ldots, 2^{a_{2}-1} q_{2} ; \cdots ; q_{m}, 2 q_{m}, \ldots, 2^{a_{m}-1} q_{m}\right\}$.

Thus such an equation has the canonical form

$$
\prod_{i=1}^{m} \frac{\left[\left(1-t^{q_{i}}\right)\left(1+t^{q_{i}}\right)\left(1+t^{2 q_{i}}\right) \ldots\left(1+t^{2^{a_{i}-1} q_{1}}\right)\right]}{1-t^{2^{a_{i} q_{i}}}}=1 \text {. }
$$

LEMMA 2. For any $n \geq 0$, there exists a diffeomorphism $f$ of the 2-dimensional unit disc onto its image with periodic data

$$
\left\{\left(2^{n+1}, 0,+1\right),(1,1,-1,),(2,1,-1),\left(2^{2}, 1,-1\right), \ldots,\left(2^{n}, 1,-1\right)\right\} .
$$

Furthermore, for some $R>0, \varepsilon>0, f$ maps the annulus $\left\{x \in \mathbf{R}^{2}|1-R<| x \mid\right.$ $<1\}$ inward along radii a fixed distance $\varepsilon$, and there exists a filtration of the disc for $f$. 
Proof of the Theorem.

THEOREM. Let $g \in \operatorname{Diff}\left(T^{2}\right)$ and suppose $g$ preserves orientation. There exists a Morse-Smale diffeomorphism $f$ on $T^{2}$ isotopic to $g$ and with periodic data $\left\{\left(p_{i}, u_{i}, \Delta_{i}\right)\right\}_{i=1}^{n}$ if and only if

(1) $u_{i}=0$ and $u_{j}=2$ for some $i$ and $j$;

(2) $\eta_{g}(t)=\prod_{i=1}^{n}\left(1-\Delta_{i} t^{P_{i}}\right)^{(-1)^{i_{i}+1}}$;

(3) $\Delta_{i}=+1$ for each $i$ with $u_{i} \in\{0,2\}$.

The necessity of conditions (1) and (3) is clear. That condition (2) is necessary is a consequence of the theorem quoted from [2]. We must show that conditions (1)-(3) together are sufficient. For a diffeomorphism to satisfy condition (2) each of its induced homology maps must be quasi-unipotent. The following lemma will be used to reduce the number of isotopy classes to be analyzed.

LEMMA 3. Let $A \in \mathrm{Gl}(2, Z)$ and suppose that both eigenvalues of $A$ are roots of unity. Then $A$ is similar over the integers to exactly one of the following matrices:

$$
\begin{gathered}
\left(\begin{array}{ll}
1 & m \\
0 & 1
\end{array}\right),\left(\begin{array}{rr}
-1 & m \\
0 & -1
\end{array}\right),\left(\begin{array}{rr}
1 & 0 \\
0 & -1
\end{array}\right),\left(\begin{array}{rr}
1 & 1 \\
0 & -1
\end{array}\right), \\
\left(\begin{array}{rr}
0 & 1 \\
-1 & 0
\end{array}\right),\left(\begin{array}{rr}
0 & 1 \\
-1 & -1
\end{array}\right),\left(\begin{array}{rr}
0 & -1 \\
1 & 1
\end{array}\right), \quad m \in\{0,1,2, \ldots\} .
\end{gathered}
$$

Proof. Let $A=\left(\begin{array}{ll}a & b \\ c & d\end{array}\right) \in \mathrm{Gl}(2, Z)$. The eigenvalues of $A$ are

$$
\frac{1}{2}\left(a+d \pm \sqrt{(a-d)^{2}+4 b c}\right) \text {. }
$$

Considering separately the cases in which $(a-d)^{2}+4 b c$ is positive and negative it is easy to show that for the eigenvalues to be roots of unity, they must be $\pm 1, \pm i,-\frac{1}{2} \pm(\sqrt{3} / 2) i$, or $\frac{1}{2} \pm(\sqrt{3} / 2) i$. Using Theorem III.13 of [7] and results in [4] it follows that any two quasi-unipotent elements of $\mathrm{Gl}(2, Z)$ with the same complex eigenvalues are similar over the integers. Since $\left(\begin{array}{rr}0 & 1 \\ -1 & 0\end{array}\right),\left(\begin{array}{rr}0 & 1 \\ -1 & -1\end{array}\right)$ and $\left(\begin{array}{c}0 \\ 1\end{array}\right)$ are representatives with eigenvalues $\pm i$, $-\frac{1}{2} \pm(\sqrt{3} / 2) i$ and $\frac{1}{2} \pm(\sqrt{3} / 2) i$ respectively, it remains to deal with the elements of $\operatorname{Gl}(2, Z)$ whose eigenvalues are \pm 1 . By Theorem III.12 in [7] it is sufficient to consider upper triangular matrices. Suppose

$$
\left(\begin{array}{ll}
x & y \\
z & w
\end{array}\right) \in \mathrm{Gl}(2, z) \text { and }\left(\begin{array}{ll}
x & y \\
z & w
\end{array}\right)\left(\begin{array}{ll}
1 & m \\
0 & 1
\end{array}\right)=\left(\begin{array}{ll}
1 & n \\
0 & 1
\end{array}\right)\left(\begin{array}{ll}
x & y \\
z & w
\end{array}\right)
$$

and $m \in Z-\{0\}$.

Equating the corresponding entries of the matrices after multiplication yields $z=0$ and $x=(n / m) w$.

Then $\pm 1=x w-y z=x w$ and thus $x, w \in\{1,-1\}$ and $m= \pm n$. Consequently any element of $\operatorname{Gl}(2, Z)$ whose eigenvalues are both 1 is similar to 
$\left(\begin{array}{ll}1 & m \\ 0 & 1\end{array}\right)$ for exactly one nonnegative integer $m$. If the eigenvalues are \pm 1 or both -1 , the proof is similar.

LEMMA 4. Let $f, g, h \in \operatorname{Diff}(M)$ with $f$ Morse-Smale and $h f=g h$. Then $g$ is Morse-Smale and the periodic data of $g$ is equal to the periodic data of $f$.

Proof. The proof that $g$ is Morse-Smale is in [1]. From that proof it follows easily that the periodic data is a differentiable conjugacy invariant.

For a diffeomorphism on $T^{2}$, the isotopy (and homotopy) class is completely determined by the action of its induced map on first homology. Since any element of $\mathrm{Gl}(2, Z)$ induces a diffeomorphism of $T^{2}=\mathbf{R}^{2} / Z^{2}$, to prove the theorem it suffices to consider the isotopy classes of maps on $T^{2}$ induced by $\left(\begin{array}{ll}1 & m \\ 0 & 1\end{array}\right),\left(\begin{array}{rr}-1 & m \\ 0 & -1\end{array}\right),\left(\begin{array}{rr}0 & 1 \\ -1 & 0\end{array}\right),\left(\begin{array}{rr}0 & 1 \\ -1 & -1\end{array}\right)$ and $\left(\begin{array}{cc}0 & -1 \\ 1\end{array}\right)$.

For ( $\left.\begin{array}{ll}1 & g \\ 0 & 1\end{array}\right)$, this is Narasimhan's theorem with $M=T^{2}$. Her construction involves performing homotopies on annuli and then connecting them together as cylinders to form a torus. The case for $\left(\begin{array}{ll}1 & m \\ 0 & 1\end{array}\right)$ is obtained by adding an additional annulus $A_{0}$ on which a diffeomorphism is defined which increases radial distances and with the image of each radial segment winding around the annulus $m$ times. This changes the isotopy class, but neither the periodic data of the map nor its homology zeta function is altered.

Let $g$ be the map on $T^{2}$ induced by $\left(\begin{array}{rr}0 & 1 \\ -1 & -1\end{array}\right)$. The homology zeta function of this map is

$$
\eta(t)=\frac{1+t+t^{2}}{(1-t)^{2}}=\frac{1-t^{3}}{(1-t)^{3}}
$$

Suppose $\left\{\left(p_{i}, u_{i}, \Delta_{i}\right)\right\}_{i=1}^{n}$ is a set of periodic data satisfying conditions (1)-(3) of the theorem. Then

$$
1=\frac{(1-t)^{3} \Pi_{i=1}^{l}\left(1-t^{q_{i}}\right) \Pi_{i=1}^{s}\left(1+t^{r_{i}}\right)}{\left(1-t^{3}\right) \Pi_{j=1}^{m}\left(1-t^{P_{j}}\right)}
$$

where the denominator product is over the sink and source orbits and the numerator products range, respectively, over the orientation preserving and reversing saddle orbits.

We now apply Lemma 1 . Since $1-t^{3}$ occurs in the denominator of the above expression, there exists $i$ (say $i=1$ ) with $q_{i}=3$ and thus the periodic data must include an orientation preserving saddle of period 3. For each of the three $1-t$ factors in the numerator, there exists an $a_{i}(i=-1,0,1)$ such that the periodic data includes $\left(2^{a_{i}}, u_{i},+1\right),(1,1,-1), \ldots$, and $\left(2^{a_{i}-1}, 1,-1\right)$ with $u_{i} \in\{0,2\}$. Applying the lemma to the remaining factors, the periodic data can be rewritten: 


$$
\begin{gathered}
P=\left\{(3,1,+1) ;\left(2^{a_{-1}}, u_{-1},+1\right),(1,1,-1), \ldots,\left(2^{a_{-1}-1}, 1,-1\right) ;\right. \\
\left(2^{a_{0}}, u_{0},+1\right),(1,1,-1), \ldots,\left(2^{a_{0}-1}, 1,-1\right) ; \\
\left(2^{a_{1}}, u_{1},+1\right),(1,1,-1), \ldots,\left(2^{a_{1}-1}, 1,-1\right) ; \\
\left.\left(2^{a_{i}} q_{i}, u_{i},+1\right),\left(q_{i}, 1,+1\right),\left(q_{i}, 1,-1\right), \ldots,\left(2^{a_{i}-1} q_{i}, 1,-1\right)\right\}, \\
i=2, \ldots, l .
\end{gathered}
$$

We must now construct a Morse-Smale diffeomorphism in the isotopy class of $g$ with this periodic data. We begin by isotoping $g$ to Morse-Smale diffeomorphisms with "minimal" periodic data. Let $\varphi$ be a diffeomorphism on $T^{2}=R^{2} / Z^{2}$ which commutes with $g$, is isotopic to the identity, and whose hyperbolic dynamics on the unit square are shown in Figure 1. Note that the triangles are preserved by $g$. Thus $f_{1}=g \circ \varphi$ and $f_{2}=g \circ \varphi^{-1}$ are Morse-Smale diffeomorphisms isotopic to $f$ with periodic data

$$
\{(3,1,+1),(1,0,+1),(1,0,+1),(1,2,+1)\}
$$

and

$$
\{(3,1,+1),(1,0,+1),(1,2,+1),(1,2,+1)\},
$$

respectively.

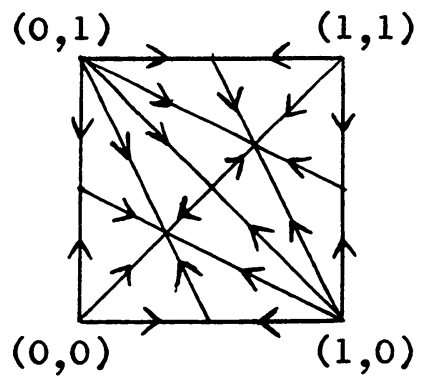

FIGURE 1

Consider the periodic data set $P$. Each of the indices $u_{-1}, u_{0}$, and $u_{1}$ equal either 0 or 2. We will assume that at least two of them (say $u_{-1}$ and $u_{0}$ ) are 0 and then isotope $f_{1}$ to a map with periodic data $P$. A similar argument with $f_{2}$ will handle the cases when there are more 2's than 0's.

For both fixed sinks of $f_{1}$ we can use Theorem 5.6 of [5] to produce a smooth isotopy on a neighborhood of the sink. The result is a diffeomorphism with the same nonwandering set but whose local representative on a smaller neighborhood of each sink takes the form $\left(\begin{array}{l}\lambda \\ 0 \\ \lambda\end{array}\right)$ with $0<\lambda<1$. Applying Lemma 2 to isotope on the smaller neighborhoods, the periodic data of $f_{1}$ can be changed from

$$
\{(3,1,+1),(1,0,+1),(1,0,+1),(1,2,+1)\}
$$


to

$$
\begin{array}{r}
Q=\left\{(3,1,+1),(1,2,+1),\left(2^{a_{-1}}, 0,+1\right),(1,1,-1), \ldots,\left(2^{a_{-1}-1}, 1,-1\right),\right. \\
\left.\left(2^{a_{0}}, 0,+1\right),(1,1,-1), \ldots,\left(2^{a_{0}-1}, 1,-1\right)\right\} .
\end{array}
$$

Call the new diffeomorphism $f_{0}$.

The product over $P-Q$ of $\left(1-\Delta_{i} t^{P_{i}}\right)^{(-1)^{m_{i+1}+1}}$ is equal to $1 /(1-t)$. By assumption the periodic data in $P-Q$ includes a source. Narasimhan's theorem yields a Morse-Smale diffeomorphism of $S^{2}$ with periodic data $(P-Q) \cup\{(1,0,+1)\}$. When a neighborhood of the sink is removed, the resulting map is a diffeomorphism of a disc onto its image with points near the boundary being mapped outside the disc. The periodic data of this map is $P-Q$. Isotoping this diffeomorphism into a neighborhood of the source in $f_{0}$ (i.e. the original source from $f_{1}$ ) yields an $\Omega$-stable diffeomorphism with periodic data $P$. Taking a Kupka-Smale approximation completes the case.

In the isotopy classes of maps induced by

the homology zeta functions are

$$
\left(\begin{array}{rr}
0 & 1 \\
-1 & 0
\end{array}\right), \quad\left(\begin{array}{rr}
0 & -1 \\
1 & 1
\end{array}\right), \quad \text { and } \quad\left(\begin{array}{rr}
-1 & m \\
0 & -1
\end{array}\right)
$$

$$
\frac{1+t^{2}}{(1-t)^{2}}, \quad \frac{1+t^{3}}{\left(1-t^{2}\right)(1-t)}, \text { and } \frac{(1+t)^{2}}{(1-t)^{2}}
$$

respectively. The following lemma is useful in determining certain minimal periodic data which occur in these isotopy classes.

LEMMA 5. If

$$
\frac{\Pi_{i=1}^{m}\left(1-t^{q_{i}}\right) \Pi_{i=1}^{n}\left(1+t^{r_{i}}\right)}{\left(1+t^{k}\right)^{s} \Pi_{j=1}^{l}\left(1-t^{p_{j}}\right)}=1
$$

then there exist nonnegative integers $s_{1}$ and $s_{2}$ with $s=s_{1}+s_{2}$ and such that

(1) at least $s_{1}$ of the $r_{i}$ are $k$;

(2) at least $s_{2}$ of the $q_{i}$ are $2 k$;

(3) at least $s_{2}$ of the $p_{j}$ are $k$.

Proof. Multiplying numerator and denominator of the expression on the left by $\left(1-t^{k}\right)^{s}$ and applying Lemma 1 to the resulting $s\left(1-t^{2 k}\right)$ factors in the denominator yields (1) and (2). Fix $s_{1}$ to be the number of the $r_{i}$ that are $k$. Cancelling $\left(1+t^{k}\right)^{s_{1}}\left(1-t^{k}\right)^{s_{1}}$ from the numerator with $\left(1-t^{2 k}\right)^{s_{1}}$ from the denominator leaves

$$
\frac{\left(1-t^{k}\right)^{s_{2}} \Pi\left(1-t^{q_{i}}\right) \Pi\left(1+t^{r_{i}}\right)}{\left(1-t^{2 k}\right)^{s_{2}} \Pi\left(1-t^{p_{j}}\right)}
$$

where no $r_{i}$ is $k$.Another application of Lemma 1 establishes (3). 
Let $g$ be the diffeomorphism on $T^{2}$ induced by $\left(\begin{array}{rl}0 & 1 \\ -1 & 0\end{array}\right)$. Let $\varphi$ be a diffeomorphism on $T^{2}=R^{2} / Z^{2}$ which commutes with $g$, is isotopic to the identity, and whose hyperbolic dynamics on the unit square are shown in Figure 2. Then $f_{1}=g \circ \varphi$ is a Morse-Smale diffeomorphism isotopic to $g$ with periodic data $\{(2,1,-1),(1,0,+1),(1,2,+1)\}$.

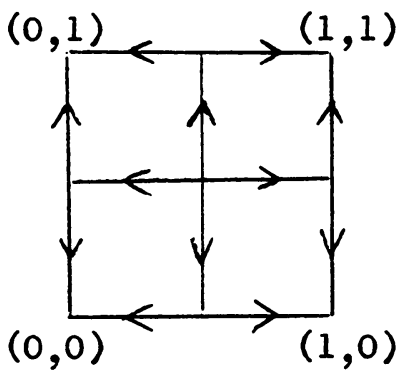

FIGURE 2

Suppose $P=\left\{\left(p_{i}, u_{i}, \Delta_{i}\right)\right\}_{i=1}^{n}$ is a set of periodic data satisfying conditions $(1)-(3)$ in the theorem. By Lemma $5, P$ contains either $(2,1,-1)$ or it contains $(4,1,+1)$ and $(2, u,+1)$ where $u$ is equal to 0 or 2 . If $(2,1,-1) \in$ $P$, then the additional data can be isotoped into neighborhoods of the source and sink in $f_{1}$.

If $(2,1,-1) \notin P$, consider the Morse-Smale diffeomorphisms isotopic to $g$ with periodic data

$$
\{(4,1,+1),(2,0,+1),(1,2,+1),(1,2,+1)\}
$$

and

$$
\{(4,1,+1),(2,2,+1),(1,2,+1),(1,0,+1)\} \text {. }
$$

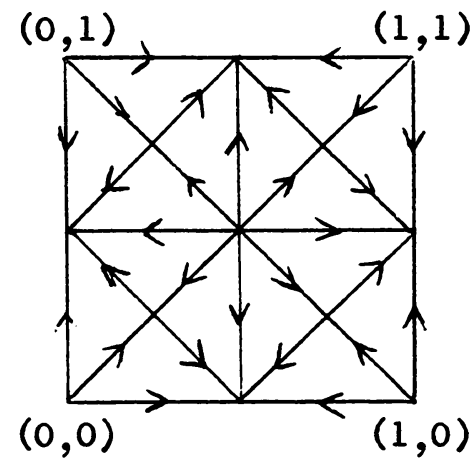

FIGURE 3

The first set is obtained by composing $g$ with the map whose dynamics are shown in Figure 3. Locally isotoping the period 2 saddle in $f_{1}$ to a period 4 
orientation preserving saddle and period 2 source yields the second data set. One can define analogous maps with the sink and source data reversed. Beginning with one of these four diffeomorphisms, isotopies in the neighborhoods of fixed points will result in the data set $P$.

To complete the proof of the theorem similar arguments can be used for the isotopy classes of the maps induced by $\left(\begin{array}{cc}0 & -1 \\ 1\end{array}\right)$ and $\left(\begin{array}{rr}-1 & m \\ 0 & -1\end{array}\right)$.

We close with two remarks on the hypothesis that $g$ be an orientation preserving diffeomorphism on the torus. John Franks has shown me an example of an orientation reversing isotopy class on $T^{2}$ and a periodic data set satisfying conditions (1) and (2) of the theorem which cannot be the periodic data set of a Morse-Smale diffeomorphism in the isotopy class. For orientable surfaces with negative Euler characteristic there exist isotopy classes which do not admit Morse-Smale diffeomorphisms but on which the induced map on first homology is the identity [9].

\section{BIBLIOGRAPHY}

1. S. Batterson, Structurally stable Grassmann transformations, Trans. Amer. Math. Soc. 231 (1977), 385-405.

2. J. Franks, Some smooth maps with infinitely many hyperbolic periodic points, Trans. Amer. Math. Soc. 226 (1977), 175-179.

3. J. Franks and C. Narasimhan, The periodic behavior of Morse-Smale diffeomorphisms, Invent. Math. 48 (1978), 279-292.

4. J. Masley and H. Montgomery, Cyclotomic fields with unique factorization, J. Reine Angew. Math. 286/287 (1976), 248-256. 1965.

5. J. Milnor, Lectures on the h-cobordism theorem, Princeton Univ. Press, Princeton, N. J.,

6. C. Narasimhan, The periodic behavior of Morse-Smale diffeomorphisms on compact surfaces, Trans. Amer. Math. Soc. 248 (1979), 145-169.

7. M. Newman, Integral matrices, Academic Press, New York, 1972.

8. J. Palis and S. Smale, Structural stability theorems, Proc. Sympos. Pure Math., vol. 14, Amer. Math. Soc., Providence, R. I., 1970, pp. 223-231.

9. L. da Rocha, Caracterizacao das classes de isotopia Morse-Smale em superficies, Thesis, IMPA, 1978.

10. M. Shub, Morse-Smale diffeomorphisms are unipotent on homology, Dynamical systems (Proc. Sympos., Univ. Bahia, Salvador, 1971), Academic Press, New York, 1973.

11. M. Shub and D. Sullivan, Homology theory and dynamical systems, Topology 14 (1975), $109-132$.

Department of Mathematics, Emory University, Atlanta, Georgin 30322 